ARTICLE

https://doi.org/10.1038/s41467-019-11761-1

\title{
Measurement of the variation of electron-to-proton mass ratio using ultracold molecules produced from laser-cooled atoms
}

\author{
J. Kobayashi (1D ${ }^{1,2}$, A. Ogino ${ }^{3}$ \& S. Inouye 4,5
}

Experimental techniques to manipulate cold molecules have seen great development in recent years. The precision measurements of cold molecules are expected to give insights into fundamental physics. Here we use a rovibrationally pure sample of ultracold $\mathrm{KRb}$ molecules to improve the measurement on the stability of electron-to-proton mass ratio $\left(\mu=\frac{m_{\mathrm{e}}}{M_{\mathrm{p}}}\right)$. The measurement is based upon a large sensitivity coefficient of the molecular spectroscopy, which utilizes a transition between a nearly degenerate pair of vibrational levels each associated with a different electronic potential. Observed limit on temporal variation of $\mu$ is $\frac{1}{\mu} \frac{d \mu}{d t}=(0.30 \pm 1.0) \times 10^{-14}$ year $^{-1}$, which is better by a factor of five compared with the most stringent laboratory molecular limits to date. Further improvements should be straightforward, because our measurement was only limited by statistical errors.

\footnotetext{
${ }^{1}$ Department of Physics, Graduate School of Science, Kyoto University, Kyoto 606-8502, Japan. ${ }^{2}$ PRESTO, Japan Science and Technology Agency, Kyoto 606-8502, Japan. ${ }^{3}$ Department of Applied Physics, University of Tokyo, Bunkyo-ku, Tokyo 113-8656, Japan. ${ }^{4}$ Graduate School of Science, Osaka City University, Osaka 558-8585, Japan. ${ }^{5}$ Nambu Yoichiro Institute of Theoretical and Experimental Physics (NITEP), Osaka City University, Osaka 558-8585, Japan. Correspondence and requests for materials should be addressed to J.K. (email: j-kobayashi@scphys.kyoto-u.ac.jp)
} 
C old molecules are becoming a popular tool for precision measurements. The search for the electron electric dipole moment $(E D M)$ is a good example ${ }^{1-3}$. Historically, hot atoms in a vapor cell or in an atomic beam were gradually replaced with cold molecular sources and trapped molecular ions. Cold molecules have a number of advantages for increasing the precision, like longer interaction time, smaller motional decoherence, and it is easier to make use of its rich internal degrees of freedom ${ }^{4}$. In the case of EDM experiments, molecules (and molecular ions) were placed in $\Omega$ doublet levels, where large internal electric field and systematic error rejection are available ${ }^{5}$. Similarly, one can expect to improve the measurement of the variation of fundamental constants by selecting molecules with a level structure that enhances the sensitivity.

Thus far, various physical systems and experimental techniques have been used to measure the stability of the electron-to-proton mass ratio $\mu=m_{\mathrm{e}} / M_{\mathrm{p}}$ : each of them provide a crucial independent check ${ }^{6,7}$. Molecular spectroscopy is often called model-free, because it directly reflects the stability of the inertial mass of the nucleus. Shelkovnikov et al. studied the absorption lines of $\mathrm{SF}_{6}$ and obtained a limit on the current variation of $\mu$ as $\frac{1}{\mu} \frac{d \mu}{d t}=(-3.8 \pm 5.6) \times 10^{-14}$ year $^{-18}$.

In the mean time, impressive results of $\frac{1}{\mu} \frac{d \mu}{d t}=$ $0.2(1.1) \times 10^{-16}$ year $^{-19}$ and $\frac{1}{\mu} \frac{d \mu}{d t}=-0.5(1.6) \times 10^{-16}$ year $^{\mu} \frac{d t}{10}$ were obtained by using atomic clocks. These measurements are essentially measuring the variation of the electron-to-proton magnetic moment ratio.

Here we report a measurement on the variation of $\mu$ using ultracold KRb molecules. Diatomic alkali molecules like KRb have two low-lying, overlapping potentials: the deep $X^{1} \Sigma^{+}$ground state and the shallower $a^{3} \Sigma^{+}$state. It has been pointed out that using a transition between a nearly degenerate pair of vibrational levels each associated with $X^{1} \Sigma^{+}$and $a^{3} \Sigma^{+}$potentials, one can realize an enhanced sensitivity to the variation of $\mu^{11}$. We realize such an enhancement (on the order of $\sim 10^{4}$ ) by using ultracold $\mathrm{KRb}$ molecules produced by photoassociation in a magnetooptical trap followed by stimulated Raman adiabatic passage ${ }^{12}$. We obtain a better result than previous measurements using molecules ${ }^{8}$. That is solely limited by counting statistics and demonstrates the power of the method.

\section{Results}

Sensitivity to the variation of $\mu$. To quantify the sensitivity of the transition to the variation of fundamental constants, we define the sensitivity $W_{\mu}$ and the sensitivity coefficient $K_{\mu}$ as:

$$
\Delta \nu=W_{\mu} \frac{\Delta \mu}{\mu}=\nu K_{\mu} \frac{\Delta \mu}{\mu} .
$$

$W_{\mu}$ and $K_{\mu}$ determine the absolute and relative accuracy needed for frequency standards used in the experiment. In general, $\left|K_{\mu}\right|$ s for vibrational and rotational transitions of diatomic molecules are $1 / 2$ and 1 , respectively. Large sensitivity coefficients can be realized using transitions between nearly degenerate states with different symmetries. Such transitions were successfully used in previously mentioned astronomical observations, where mixed torsion-rotation transitions with $K_{\mu}=-32.8$ were investigated ${ }^{13}$. A similar idea was used to improve the limit on the variation of the fine-structure constant ${ }^{14}$.

Target transition. In this study, we used such transitions in ultracold $\mathrm{KRb}$ molecules. As pointed out in ref. ${ }^{11}$, closely lying states in $X^{1} \Sigma^{+}$and $a^{3} \Sigma^{+}$potentials give rise to a large $K_{\mu}$. Figure 1 shows the actual energy curves calculated for ${ }^{41} \mathrm{~K}^{87} \mathrm{Rb}^{15}$. From these potential energy curves we can calculate the sensitivity for each vibrational state with the accuracy only being limited by the uncertainty of the potential energy curve ("Methods" section). Although the vibrational states with the binding energies about $1000 \mathrm{~cm}^{-1}$ in $X^{1} \Sigma^{+}$have the highest sensitivities of $W_{\mu}=35 \mathrm{THz}$, their energies are far from that of the vibrational states in $a^{3} \Sigma^{+}$, whose largest binding energy is about $240 \mathrm{~cm}^{-1}$.

We found that one of the best combinations is that of the $v=$ 86 state in the $X^{1} \Sigma^{+}$potential and the $v=16$ state in the $a^{3} \Sigma^{+}$ potential because the transition frequency is in the microwave range, the sensitivity coefficient is large $\left(\sim 10^{4}\right)$, and the strength of the transition is not too small owing to the finite singlet-triplet mixing $(\sim 2 \%)$. Though its sensitivity is not the highest, it is as high as about one fourth of that. Moreover, it is another advantage that the experiments with microwaves are easier than the experiments with frequencies in the optical domain.

Understanding the details of the closely lying hyperfine manifolds is mandatory for estimating the strength of each microwave transition. In the following discussion, we express the hyperfine states as $\left|S, F_{1}, F, m_{F}\right\rangle$ in short, where the detail of labeling is in the "Method" section. Note that $S=0 \quad(S=1)$ signifies the $v=86$ state in $X^{1} \Sigma^{+}\left(v=16\right.$ state in $\left.a^{3} \Sigma^{+}\right)$.

We obtained the hyperfine spectra by sweeping the frequency of the stimulated Raman adiabatic passage (STIRAP) laser (Fig. 2). The results are summarized in the Supplementary Note 1 and Supplementary Table 1 . We identified the most suitable transition to test the stability of $\mu$ as $|\mathrm{i}\rangle-|\mathrm{f}\rangle$ (Fig. 1) where

$$
\begin{aligned}
& |\mathrm{i}\rangle \equiv\left|S=0, F_{1}=3 / 2, F=0, m_{F}=0\right\rangle, \\
& |\mathrm{f}\rangle \equiv\left|S=1, F_{1}=1 / 2, F=1, m_{F}=0\right\rangle .
\end{aligned}
$$

The $|i\rangle-|f\rangle$ transition fulfills the required conditions: the sensitivity to the variation of $\mu$ is large, the magnetic sublevels are resolved by a small magnetic field, and there is no first-order Zeeman shift. The transition frequency is $v_{\mathrm{i}-f}=634.96 \mathrm{MHz}$, and the sensitivity of the transition to the variation of $\mu$ is $W_{\mathrm{i}-f}=\partial v_{\mathrm{i}}$ ${ }_{-f} / \partial(\ln \mu)=-9.45(4) \mathrm{THz}$ ("Methods" section). The resulting sensitivity coefficient is:

$$
K_{\mu, \mathrm{i}-f}=\frac{W_{\mathrm{i}-f}}{\nu_{\mathrm{i}-f}}=-14890(60) .
$$

Spectroscopy. Figure 3 shows a typical spectrum of the $|i\rangle-|f\rangle$ transition obtained by $6 \mathrm{~h}$ of data collection. A Gaussian fit to the data provided the full-width-of-half-maximum (FWHM) of $\sim 50 \mathrm{~Hz}$, which is consistent with the ideal spectrum obtained by the microwave $\pi$-pulse of $16 \mathrm{~ms}$ duration. Longer pulse durations degraded the signal because ballistically expanding clouds of molecules with mean velocity $\sim 130 \mathrm{~mm} \mathrm{~s}^{-1}$ started to leave the area of the ionization beam whose radius was $\sim 2 \mathrm{~mm}$. To access only the $m_{F}=0$ state in the $\left|S=1, F_{1}=1 / 2, F=1\right\rangle$ manifold a magnetic field of $\sim 1.5 \mu \mathrm{T}$ was applied. The measurements were repeated every $120 \mathrm{~ms}$. The statistical uncertainty of the central frequency was $\sim 93 \mathrm{mHz}$.

Statistical and systematic errors. We paid special attention to the magnetic field during the measurement as it drifts because of the magnetization of the metal chamber and fluctuates due to other environmental factors. The $|\mathrm{i}\rangle-|\mathrm{f}\rangle$ transition is immune to the first-order Zeeman shift; however, it is affected by the second-order Zeeman shift $\left(\sim 0.82 \mathrm{~Hz} \mu \mathrm{T}^{-2}\right)$. To evaluate this shift, we simultaneously measured the transition frequency to the hyperfine state $\left|f_{1}\right\rangle \equiv\left|S=1, F_{1}=1 / 2, F=1, m_{F}=1\right\rangle$ whose energy is sensitive to the first-order Zeeman shift $\left(\sim 3.3 \mathrm{kHz} \mu \mathrm{T}^{-1}\right)$. The observations for $|i\rangle-|\mathrm{f}\rangle$ and $|\mathrm{i}\rangle-\left|\mathrm{f}_{1}\right\rangle$ alternated every $2.4 \mathrm{~s}$, and the frequency of the $|i\rangle-\left|f_{1}\right\rangle$ transition was used to subtract the second-order Zeeman shift from the measured $|i\rangle-|f\rangle$ transition frequencies. 


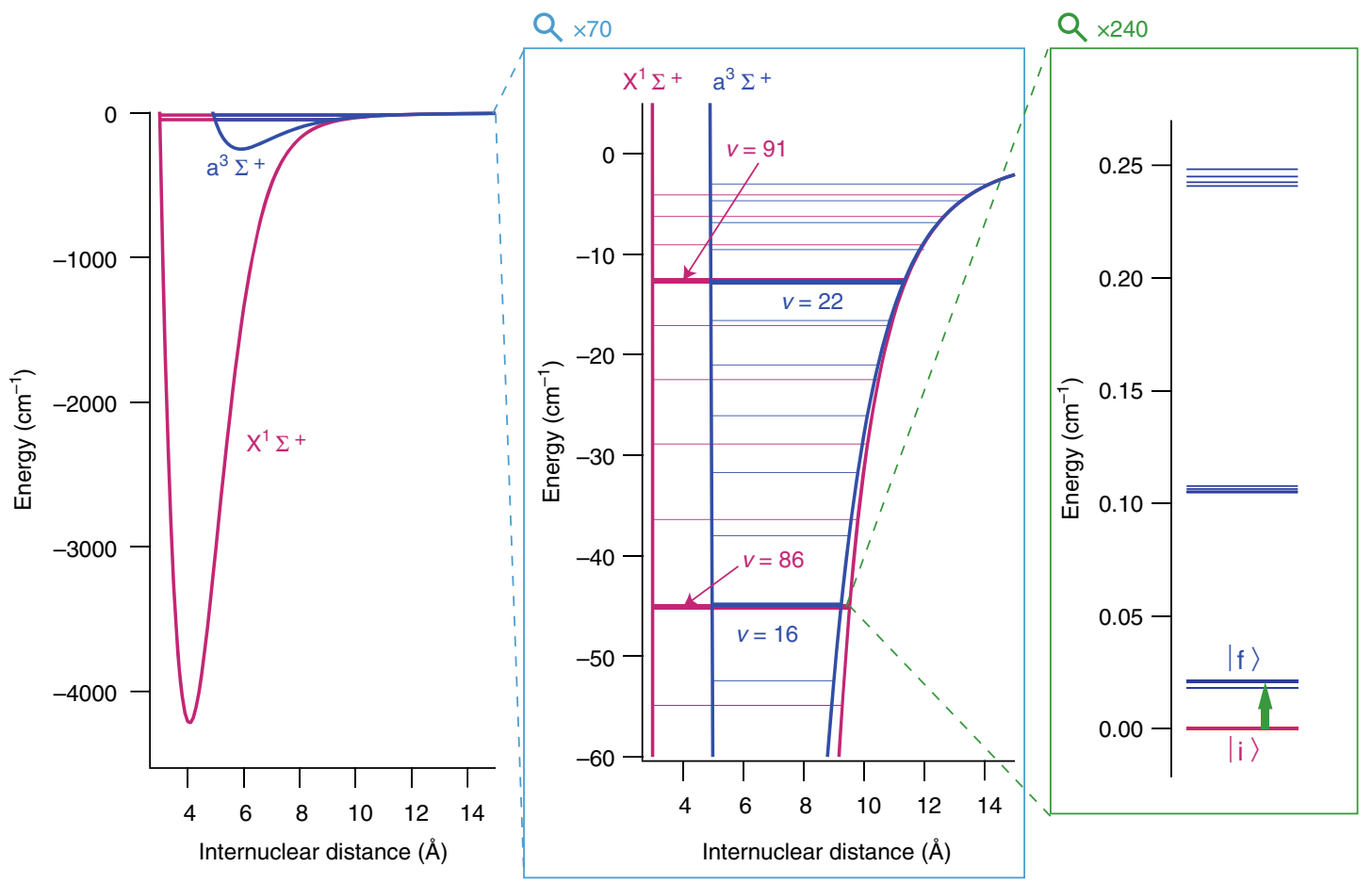

Fig. 1 Closely lying states of a ${ }^{41} \mathrm{~K}^{87} \mathrm{Rb}$ molecule ${ }^{15}$. In the shallow range of the potentials, several pairs of closely lying vibrational states in $X^{1} \Sigma^{+}$and $a^{3} \Sigma^{+}$ potentials are found. Among such pairs, we selected the combination of $v=86$ state in $X^{1} \Sigma^{+}$and $v=16$ state in $a^{3} \Sigma^{+}$. The large difference of potential depth between $X^{1} \Sigma^{+}$and $a^{3} \Sigma^{+}$leads to the large sensitivity $W \equiv \partial \nu / \partial(\ln \mu) \approx-9.5 \mathrm{THz}$, whereas the transition frequency itself ( $\equiv \nu$ ) is in the microwave range $(\approx 635 \mathrm{MHz})$. This yields a large sensitivity coefficient $\left|K_{\mu}\right| \equiv|W / \nu| \approx 15,000$

The statistical and systematic errors caused by the correction step were evaluated as 15 and $1 \mathrm{mHz}$, respectively, where the systematic error is caused by the error of the Zeeman coefficients.

The blackbody radiation (BBR) shift can fluctuate owing to the temperature fluctuation of the vacuum chamber $\left(26.8 \pm 2{ }^{\circ} \mathrm{C}\right)$. The coefficient was calculated using ab initio calculations for the transition dipole moments ${ }^{16}$. The BBR shifts for $|\mathrm{i}\rangle$ and $|\mathrm{f}\rangle$ states are on the order of $1 \mathrm{~Hz}$, but the effect on the transition frequency is far smaller since two shifts almost cancel out. Because of the difficulty of the calculation, the uncertainty of the BBR shift on the transition frequency is larger than the central value of the estimation, where the BBR shifts induced by the coupling with other vibrational states in $X^{1} \Sigma^{+}$and $a^{3} \Sigma^{+}$are much smaller than that induced by the coupling with other electronic states. From both of the temperature fluctuation and the calculated BBR coefficients with the uncertainty $(0.08(20) \mathrm{Hz}$ at $300 \mathrm{~K})$, we estimated the fluctuation of the blackbody shift during the measurements as $<10 \mathrm{mHz}$.

There are several systematic effects with fluctuations $<1 \mathrm{mHz}$; hence, we can neglect them in the uncertainty budget table (Table 1). The list includes the Stark shift, the density shift, and the reference clock. The Stark shift is caused by a static electric field in the vacuum chamber, which effectively shields environmental electric field fluctuations. The main source of the electric field in the detection region is the microchannel plate (MCP). We canceled this field during the measurements by applying a compensation field from electrodes placed inside the chamber, where we measured the electric field by observing the microwave transition between the rotational states of $\left|X^{1} \Sigma^{+}, v=0, N=0\right\rangle$ and $|N=1\rangle$, whose sensitivity to the electric field $\left(\sim 12.4 \mathrm{~Hz} \mathrm{~cm}^{2}\right.$ $\left.\mathrm{V}^{-2}\right)$ is one order higher than that of the $|\mathrm{i}\rangle-|\mathrm{f}\rangle$ transition $\left(\sim 1.08 \mathrm{~Hz} \mathrm{~cm}^{2} \mathrm{~V}^{-2}\right)$. However, a finite residual electric field of $0.19(6) \mathrm{V} \mathrm{cm}^{-1}$ persists and it causes a dc-Stark shift of $\sim 40 \mathrm{mHz}$, where the stark shift coefficients are estimated by using the $a b$ initio calculation for the dipole moment ${ }^{17}$. This issue, however, is not important because the fluctuation of the Stark shift, which comes from the fluctuation of the MCP voltage, should be $<1 \mathrm{mHz}$. The fluctuation of the frequency clock is also negligible because we use a commercial Rb-frequency standard (RSR FS725) locked to the Global Positioning System signal $\left(\delta f / f \sim 10^{-12}\right)$. The density shift is also $<1 \mathrm{mHz}$ because we blast off the atomic clouds by shining the resonant lasers before the microwave application. Note that though the photoassociation recoil induces a finite mean velocity to the molecular cloud and that causes a frequency shift of about $10 \mathrm{mHz}$ at most by the Doppler effect for microwave, the shift does not affect the measurement of the temporal variation of $\mu$, because all measurements were performed in the identical configuration and the shift is also identical for all measurements.

Stability of $\boldsymbol{\mu}$. We accumulated data intermittently for about 16 months and present the result in Fig. 4. From the linear fit to the data, we obtain the temporal variation of $f$ as:

$$
\frac{1}{f} \frac{d f}{d t}=\left(-0.44 \pm 1.47_{\text {stat }} \pm 0.24_{\text {syst }}\right) \times 10^{-10} \text { year }^{-1}
$$

and the temporal variation of $\mu$ as:

$$
\frac{1}{\mu} \frac{d \mu}{d t}=\left(0.30 \pm 1.00_{\text {stat }} \pm 0.16_{\text {syst }}\right) \times 10^{-14} \text { year }^{-1} \text {. }
$$

This is the most accurate molecular test for the temporal variation of $\mu$ to date. In a precise discussion, we measured the frequency ratio of $f / f_{\mathrm{Cs}}$, where the $f_{\mathrm{Cs}}$ is the clock transition frequency of $\mathrm{Cs}$ atom. However, since the precision of our measurement is about $10^{-10}$, which is much lower than that of the other stability tests using $f_{\mathrm{Cs}}$, we ignored the shift of $f_{\mathrm{Cs}}$ for simplicity. 

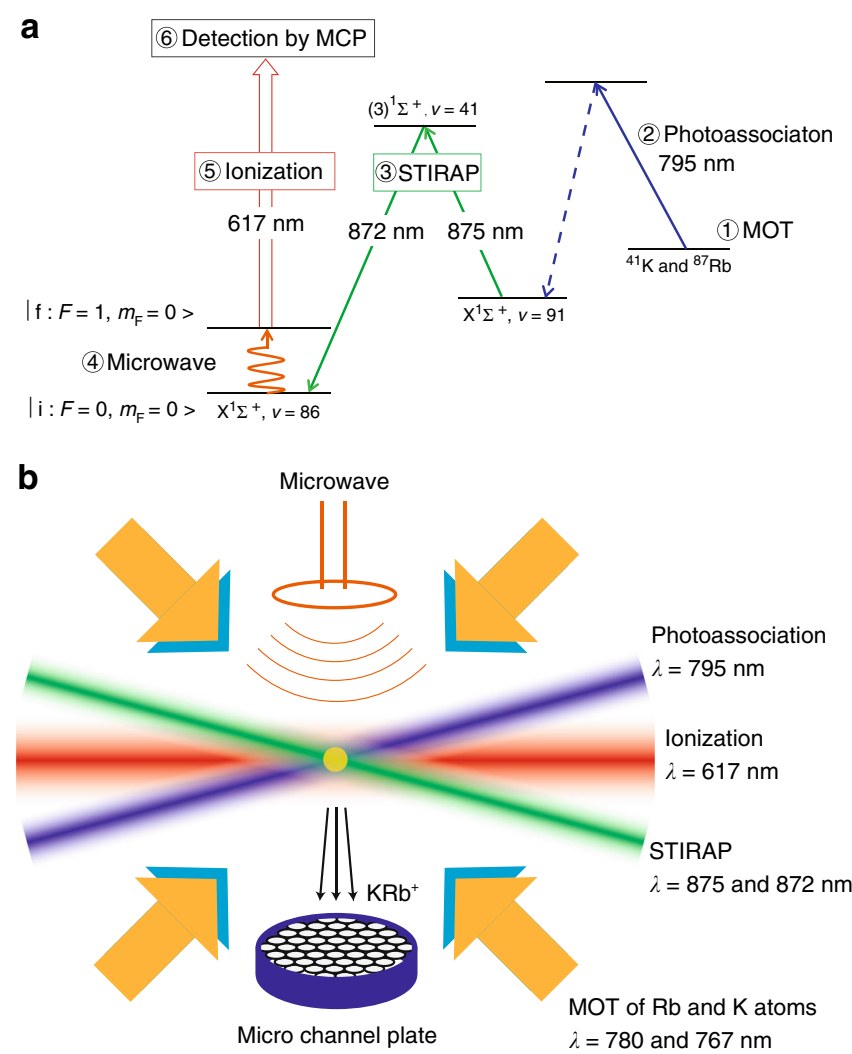

Fig. 2 Production and detection of ultracold molecules. First, ultracold atoms in a dual-species magneto-optical trap (MOT) were photoassociated into a weakly bound state. The molecules were transferred to the target internal state by stimulated Raman adiabatic passage (STIRAP) and irradiated with a microwave pulse. State selective detection of the molecules was achieved by ionizing the molecules with a pulsed laser and detecting them with a microchannel plate (MCP)

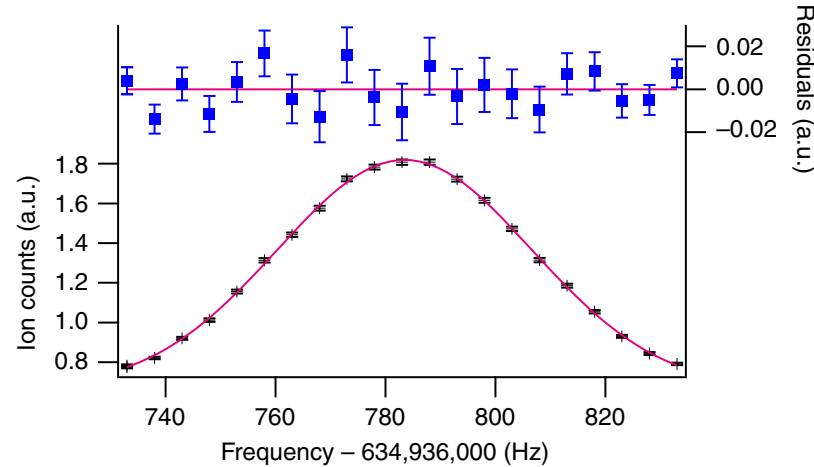

Fig. 3 Typical spectrum of the $|i\rangle-|f\rangle$ transition. Average of the ion signals and its standard deviations of mean (shown in black) are plotted against the microwave frequency. The total number of original data points used to make this plot is $\sim 95,000$, which corresponds to $6 \mathrm{~h}$ of data collection. To find the central frequency, the spectrum is fitted to a Gaussian function (magenta curve). Subtracting the Gaussian function from the ion counts gives the clear view of the residuals. The full-width-of-half-maximum (FWHM) of the spectrum is about $50 \mathrm{~Hz}$, which is limited by the microwave pulse duration of $16 \mathrm{~ms}$

\section{Discussion}

We have derived a molecular limit on the temporal variation of $\mu$ by observing the microwave transition of photoassociated KRb
Table 1 Error budget table for the measurement in Fig. 3

\section{Statistical Uncertainty}

\begin{tabular}{ll}
\hline$|i\rangle-|\mathrm{f}\rangle$ transition & $93 \mathrm{mHz}$ \\
nd-order Zeeman & $15 \mathrm{mHz}$
\end{tabular}

Statistical total $\quad 94 \mathrm{mHz}$

Systematic Fluctuation

nd-order Zeeman $1 \mathrm{mHz}$

Blackbody radiation $\quad 10 \mathrm{mHz}$

Stark $\quad 1 \mathrm{mHz}$

Reference clock $\quad<1 \mathrm{mHz}$

Density shift $\quad<1 \mathrm{mHz}$

Systematic total $\quad 10 \mathrm{mHz}$

The total uncertainty is dominated by the statistical error of the $|i\rangle-|f\rangle$ transition measurements

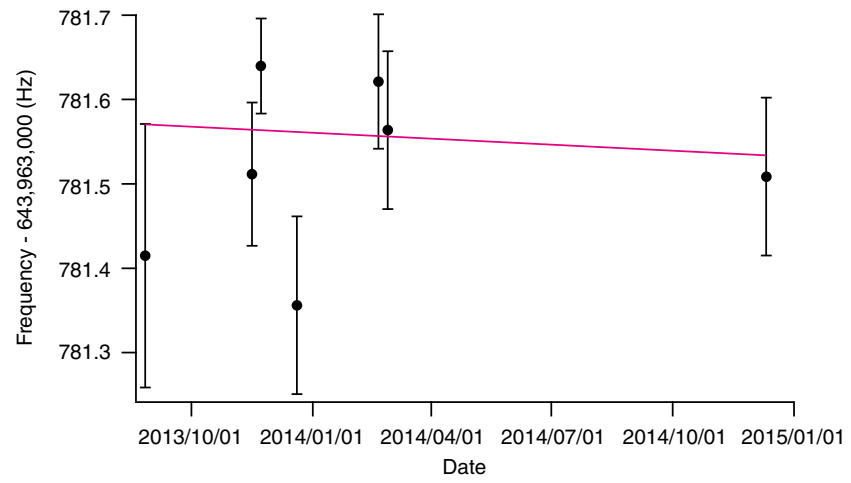

Fig. 4 Measurement of the temporal variation of $\mu$. The resonance frequencies of the $|i\rangle-|f\rangle$ transition are plotted for compensated Zeeman shifts. The error bars denote statistical standard deviations of mean. The magenta line shows a linear fit to the data with statistical errors. We obtained the temporal variation of the transition frequency $f$ : $\frac{1}{f} \frac{d f}{d t}=\left(-0.44 \pm 1.47_{\text {stat }} \pm 0.24_{\text {syst }}\right) \times 10^{-10}$ year $^{-1}$

molecules. The result is noteworthy for the following reasons. First, because of the large sensitivity coefficient of about $10^{4}$ the variation of $\mu$ was evaluated with four orders of magnitude higher accuracy than that of the frequency in our measurement. This can be a great benefit to the improvement of the precision spectroscopy because we can greatly reduce the necessity of the precision of the reference clock. Second, the sensitivities to the variation of $\mu$ of the molecular levels can be calculated from the experimentally determined molecular energy curves, thus they can be determined almost model independently. Improving the measurements using the molecular levels is important because it is independent of the measurement related to the atomic nuclear magnetic moments. Third, since ultracold molecules synthesized from ultracold atoms are used, the application to the next generation of spectroscopic methods is expected. We started to construct a machine to incorporate the idea in ref. 18 to cool alkali-metal dimers to sub-microkelvin temperatures by narrowline laser cooling. We can make the best use of sub-microkelvin molecules by building a molecular fountain setup ${ }^{11}$, where narrowing the spectral linewidth to about $1 \mathrm{~Hz}$ is expected. Another solution to achieve a narrower linewidth is holding the molecules in an optical lattice with a magic wavelength ${ }^{19}$.

By means of these techniques, spectral linewidths of about $1 \mathrm{~Hz}$ will be achieved. In addition, by making the efforts for a larger number of molecules and more data accumulations, the improvement of the precision by two-three orders of magnitude 
is expected in future experiments. Though the uncertainty of the calculation of BBR shift gives the biggest systematic error in our results, the BBR shift coefficients can be experimentally determined with good accuracy by the measurements in the longwavelength infrared fields like $\mathrm{CO}_{2}$ lasers, as performed for the ion clock transition ${ }^{20}$.

\section{Methods}

Ultracold molecular spectroscopy. Our experimental procedure for producing $\mathrm{KRb}$ molecules is described in detail in ${ }^{12}$ (Fig. 2). Briefly, KRb molecules in the $\mid X^{1} \Sigma^{+}$, $v=91, N=0\rangle$ state were produced by photoassociation in a magneto-optical trap (MOT) of ${ }^{87} \mathrm{Rb}$ and ${ }^{41} \mathrm{~K}$ atoms. The temperature of the molecules was about $140 \mu \mathrm{K}$, which is close to the temperature of atoms. Then the MOT field is turned off and both the $\mathrm{Rb}$ and $\mathrm{K}$ atoms were blasted off by shining the resonant lasers in order to suppress the energy shift caused by the molecule-atom collisions. After that, molecules were transferred to the initial state (i.e., $v=86$ state in the $X^{1} \Sigma^{+}$potential) by STIRAP. We used an intermediate state for the Raman transition of $\left|(3)^{1} \Sigma^{+}, v=41, J=1\right\rangle$ with Raman laser wavelengths of 875 and $872 \mathrm{~nm}$. After irradiating the molecules with a microwave pulse, molecules in the initial state were optically pumped to different levels by applying the $872 \mathrm{~nm}$ laser. Then the molecules in the final state were selectively observed by resonance enhanced multiphoton ionization by applying a pulsed laser whose wavenumber was $16218.1 \mathrm{~cm}^{-1}$.

Labeling of the hyperfine states. We express the hyperfine states based on Hund's case $b_{\beta S}{ }^{21}$. The hyperfine states were labeled $\left|S, I_{\mathrm{Rb}}, F_{1}, I_{\mathrm{K}}, F_{2}, N, F, m_{F}\right\rangle$, where $S$ is the total electron spin, $I_{\mathrm{Rb}}\left(I_{\mathrm{K}}\right)$ is the nuclear spin of rubidium (potassium), $N$ is the rotational angular momentum, and $F\left(m_{F}\right)$ is (the $z$ component of) the total angular momentum of the molecule. $F_{1}$ and $F_{2}$ are defined as $\mathbf{F}_{1} \equiv \mathbf{S}+\mathbf{I}_{\mathrm{Rb}}$ and $\mathbf{F}_{2} \equiv \mathbf{F}_{1}+\mathbf{I}_{\mathrm{K}}$, and $F$ satisfies the relation $\mathbf{F} \equiv \mathbf{F}_{2}+\mathbf{N}$. Note that $I_{\mathrm{Rb}}=I_{\mathrm{K}}=3 / 2$. We only discuss $N=0$ states in this letter; thus, $F=F_{2}$. In the "Results" section, we express the hyperfine states as $\left|S, F_{1}, F, m_{F}\right\rangle$ in short.

Uncertainty of the sensitivity. We calculated the sensitivities of $|i\rangle$ and $|f\rangle$ by using the experimentally determined potential energy curves reported in ${ }^{15}$. Their uncertainties were estimated from the uncertainties of the vibrational level intervals $^{11}$, which were estimated from the difference between the vibrational energies predicted by ${ }^{15}$ and our experimental observations.

\section{Data availability}

The data that support the plots within this paper and other findings of this study are available from the corresponding author upon reasonable request.

Received: 3 August 2015 Accepted: 2 August 2019

Published online: 21 August 2019

\section{References}

1. The ACME Collaboration. Improved limit on the electric dipole moment of the electron. Nature 562, 355-360 (2018)

2. Cairncross, W. B. et al. Precision measurement of the electron's electric dipole moment using trapped molecular ions. Phys. Rev. Lett. 119, 153001 (2017).

3. Hudson, J. J. et al. Improved measurement of the shape of the electron. Nature 473, 493 (2011).

4. Carr, L. D., DeMille, D., Krems, R. V. \& Ye, J. Cold and ultracold molecules: science, technology and applications. New J. Phys. 11, 055049 (2009).

5. Eckel, S., Hamilton, P., Kirilov, E., Smith, H. W. \& DeMille, D. Search for the electron electric dipole moment using $\Omega$-doublet levels in PbO. Phys. Rev. A 87, 052130 (2013).

6. DeMille, D., Doyle, J. M. \& Sushkov, A. O. Probing the frontiers of particle physics with tabletop-scale experiments. Science 357, 990-994 (2017).

7. Uzan, J.-P. The fundamental constants and their variation: observational and theoretical status. Rev. Mod. Phys. 75, 403-455 (2003).

8. Shelkovnikov, A., Butcher, R. J., Chardonnet, C. \& Amy-Klein, A. Stability of the proton-to-electron mass ratio. Phys. Rev. Lett. 100, 150801 (2008).

9. Godun, R. M. et al. Frequency ratio of two optical clock transitions in ${ }^{171} \mathrm{Yb}^{+}$ and constraints on the Time variation of fundamental constants. Phys. Rev. Lett. 113, 210801 (2014).
10. Huntemann, N. et al. Improved limit on a temporal variation of $m_{p} / m_{e}$ from comparisons of $\mathrm{Yb}^{+}$and Cs atomic clocks. Phys. Rev. Lett. 113, 210802 (2014).

11. DeMille, D. et al. Enhanced sensitivity to variation of $m_{e} / m_{p}$ in molecular spectra. Phys. Rev. Lett. 100, 043202 (2008).

12. Aikawa, K. et al. Coherent transfer of photoassociated molecules into the rovibrational ground state. Phys. Rev. Lett. 105, 203001 (2010).

13. Bagdonaite, J. et al. A stringent limit on a drifting proton-to-electron mass ratio from alcohol in the early universe. Science 339, 46-48 (2013).

14. Leefer, N., Weber, C. T. M., Cingöz, A., Torgerson, J. R. \& Budker, D. New limits on variation of the fine-structure constant using atomic dysprosium. Phys. Rev. Lett. 111, 060801 (2013).

15. Pashov, A. et al. Coupling of the $X^{1} \Sigma^{+}$and $a^{3} \Sigma^{+}$states of KRb. Phys. Rev. A 76, 022511 (2007)

16. Beuc, R. et al. Predictions for the observation of KRb spectra under cold conditions. J. Phys. B 39, S1191 (2006).

17. Kotochigova, S., Julienne, P. S. \& Tiesinga, E. Ab initio calculation of $\mathrm{KRb}$ dipole moments. Phys. Rev. A 68, 022501 (2003).

18. Kobayashi, J., Aikawa, K., Oasa, K. \& Inouye, S. Prospects for narrow-line cooling of KRb molecules in the rovibrational ground state. Phys. Rev. A 89 , 021401 (2014)

19. Zelevinsky, T., Kotochigova, S. \& Ye, J. Precision test of mass-ratio variation with lattice-confined ultracold molecules. Phys. Rev. Lett. 100, 043201 (2008).

20. Arnold, K. J., Kaewuam, R., Roy, A., Tan, T. R. \& Barrett, M. D. Blackbody radiation shift assessment for lutetium in clock. Nat. Comm. 9, 1650 (2018)

21. Townes, C. H. \& Schawlow, A. L. Microwave Spectroscopy. (Dover Publications, New York, 1975).

\section{Acknowledgements}

This work was supported by a Grant-in-Aid for Young Scientists (A) of JSPS (No. 23684034), a Grant-in-Aid for Scientific Research on Innovative Areas of JSPS (No. 24104702), JST PRESTO Grant Number JPMJPR17P5, Japan and the Matsuo Foundation.

\section{Author contributions}

J.K. and A.O. performed the experiment and analyzed the data. J.K. and S.I. supervised the project and prepared the paper.

\section{Additional information}

Supplementary Information accompanies this paper at https://doi.org/10.1038/s41467019-11761-1.

Competing interests: The authors declare no competing interests.

Reprints and permission information is available online at http://npg.nature.com/ reprintsandpermissions/

Peer review information: Nature Communications would like to thank Colin Parker, Marianna Safronova, and other anonymous reviewers for their contributions to the peer review of this work.

Publisher's note: Springer Nature remains neutral with regard to jurisdictional claims in published maps and institutional affiliations.

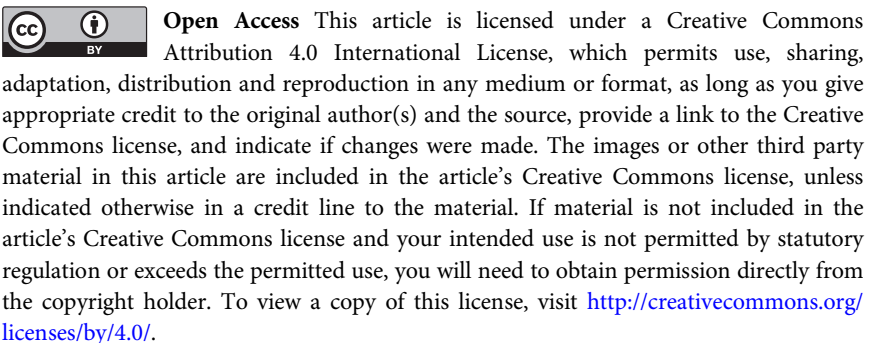

(c) The Author(s) 2019 